\title{
Brain-Machine Interfaces for Motor Control: A Guide for Neuroscience Clinicians
}

\author{
Allan R. Martin, Tejas Sankar, Nir Lipsman, Andres M. Lozano
}

\begin{abstract}
With the growing interdependence between medicine and technology, the prospect of connecting machines to the human brain is rapidly being realized. The field of neuroprosthetics is transitioning from the proof of concept stage to the development of advanced clinical treatments. In one area of brain-machine interfaces (BMIs) related to the motor system, also termed "motor neuroprosthetics', research successes with implanted microelectrodes in animals have demonstrated immense potential for restoring motor deficits. Early human trials have also begun, with some success but also highlighting several technical challenges. Here we review the concepts and anatomy underlying motor BMI designs, review their early use in clinical applications, and offer a framework to evaluate these technologies in order to predict their eventual clinical utility. Ultimately, we hope to help neuroscience clinicians understand and participate in this burgeoning field.
\end{abstract}

RÉSUMÉ: Guide sur les interface cerveau-machine pour le contrôle moteur destiné aux cliniciens en neurosciences. Étant donné l'interdépendance croissante de la médecine et de la technologie, la perspective de brancher des machines au cerveau humain se réalise rapidement. Le domaine de la neuroprothétique évolue maintenant de l'étape de la validation du principe à celui du développement de traitements cliniques de pointe. Dans le domaine des interfaces cerveau-machine (ICM) pour le système moteur, aussi connu sous le nom de "neuroprothétique motrice", les succès de la recherche sur les microélectrodes implantées chez des animaux ont démontré un potentiel immense pour pallier aux déficits moteurs. Des essais préliminaires chez les humains ont remporté un certain succès, mais ils ont aussi mis en évidence plusieurs défis techniques. Nous revoyons ici les concepts et l'anatomie pertinents à la conception des ICM motrices et leur utilisation préliminaire en clinique et nous offrons un cadre pour évaluer ces technologies afin de prédire leur utilité clinique éventuelle. Nous espérons enfin aider les cliniciens en neurosciences à comprendre et à participer à ce domaine en plein essor.

Can. J. Neurol. Sci. 2012; 39: 11-22

\section{1) Introduction}

Brain-machine interfaces (BMIs) have been defined as devices that detect intent-typically intended movement-from brain activity, and translate it into an output action, such as control of a cursor on a screen or a robotic arm. There is no doubt that the potential of BMI technology to augment normal motor performance is vast, a fact which has attracted both attention and funding from a variety of sources including United States military. ${ }^{1-3}$ For clinicians in the neurosciences, however, BMI technology could have the potential to assist in motor recovery or maintenance. At present, several forms of BMI devices, in varying stages of development, are being investigated and used to treat conditions that cause severe motor impairments, including amyotrophic lateral sclerosis (ALS), stroke, and spinal cord injury. ${ }^{4-9}$ The preliminary experiences of several groups with motor BMI applications in clinical settings have generated justifiable enthusiasm about the promise of future BMI applications. Such enthusiasm, however, is tempered by a definite need for further advances in a number of specific aspects of BMI technology. Most of these advances relate to the acquisition and long-term maintenance of high quality recordings from the brain that faithfully represent intended motor activity. Clinicians have a clear role to play not only in the neurosurgical implantation of signal recording technologies, but also in the interpretation of recorded data as it relates to motor behaviour. Furthermore, clinicians will need to determine which patient populations will benefit from implantation of these devices and under what circumstances.

Several reviews and at least one book have provided detailed descriptions of BMI technology and its potential

From the Division of Neurosurgery, Toronto Western Hospital, University Health Network, University of Toronto, Toronto, Ontario, Canada.

Received May 30, 2011. Final Revisions Submitted August 18, 2011 Correspondence to: Andres Lozano, Toronto Western Hospital, University Health Network, University of Toronto, 399 Bathurst St, 4W-447, Toronto, Ontario, M5T 2S8, Canada. 
applications. ${ }^{2,6,10-30}$ Here we provide a practical guide to BMIs for clinicians in the neurosciences. We begin by briefly summarizing the components of BMI systems. We then discuss the various approaches to neural signal acquisition, followed by a discussion of potential anatomic signal sources. Next, we review the currently available clinical data, and attempt to predict the clinical utility of BMIs in the near future. Finally, we propose criteria for evaluating BMI designs in an effort to prepare clinicians for the exciting and challenging developments that lie ahead.

\section{2) Fundamental Design of BMI Systems for Motor Control}

Any BMI designed for motor control must include three components: 1) acquiring a neural signal that can be consciously controlled; 2) analyzing that signal to identify an intended motor output; and 3) executing the intended action (Figure 1). ${ }^{6,17}$ Signal acquisition is the fundamental design-point in a BMI, since the quality and content of acquired information affects all downstream components. The nature of the acquired signal is variable, running the gamut from minimally invasive scalp electroencephalogram (EEG) data, to signals acquired through invasive neurosurgical intervention, such as intraparenchymal single neuron recordings or local field potentials. The next step is signal analysis, a complex process of identifying the user's intent and translating it into machine commands. This typically involves numerous mathematical techniques depending on the type of input signal, such as bandpower analysis after Fourier transform for an EEG signal or modeling single neuron firing rates using cosine tuning in preferred directions. ${ }^{31-33}$ In the output step, motor execution may range from the operation of a simple open-close grasper to the control of a more complex device like a multi-dimensional robotic arm. Some have even imagined sophisticated systems like full-body mechanized exoskeletons or the reanimation of paralyzed limbs with functional electrical stimulation (FES). ${ }^{34-39}$ Of particular relevance to neuroscience clinicians is the possibility that FES may evolve sufficiently to enable natural, coordinated movement, necessitating, for example, considerable neurosurgical expertise to ensure the safe implantation of integrated BMI/FES systems. In such a scenario, physicians with expertise in physiatry and rehabilitation medicine will play a central role in monitoring patients as they use FES to recover from motor deficits.

In addition to the three fundamental components of a BMI system listed above, some form of a feedback mechanism is also essential. Often visual, feedback allows both the human and machine to make continuous adjustments as the desired target is approached. Feedback is also necessary for longer-term adjustments, which may include both human and machine adaptation. Neuroplasticity, which may range from individual synaptic changes to modulation of high-level circuits, depends upon such feedback and is known to occur after hours of training. ${ }^{40-43}$ However, the underlying mechanisms for much of

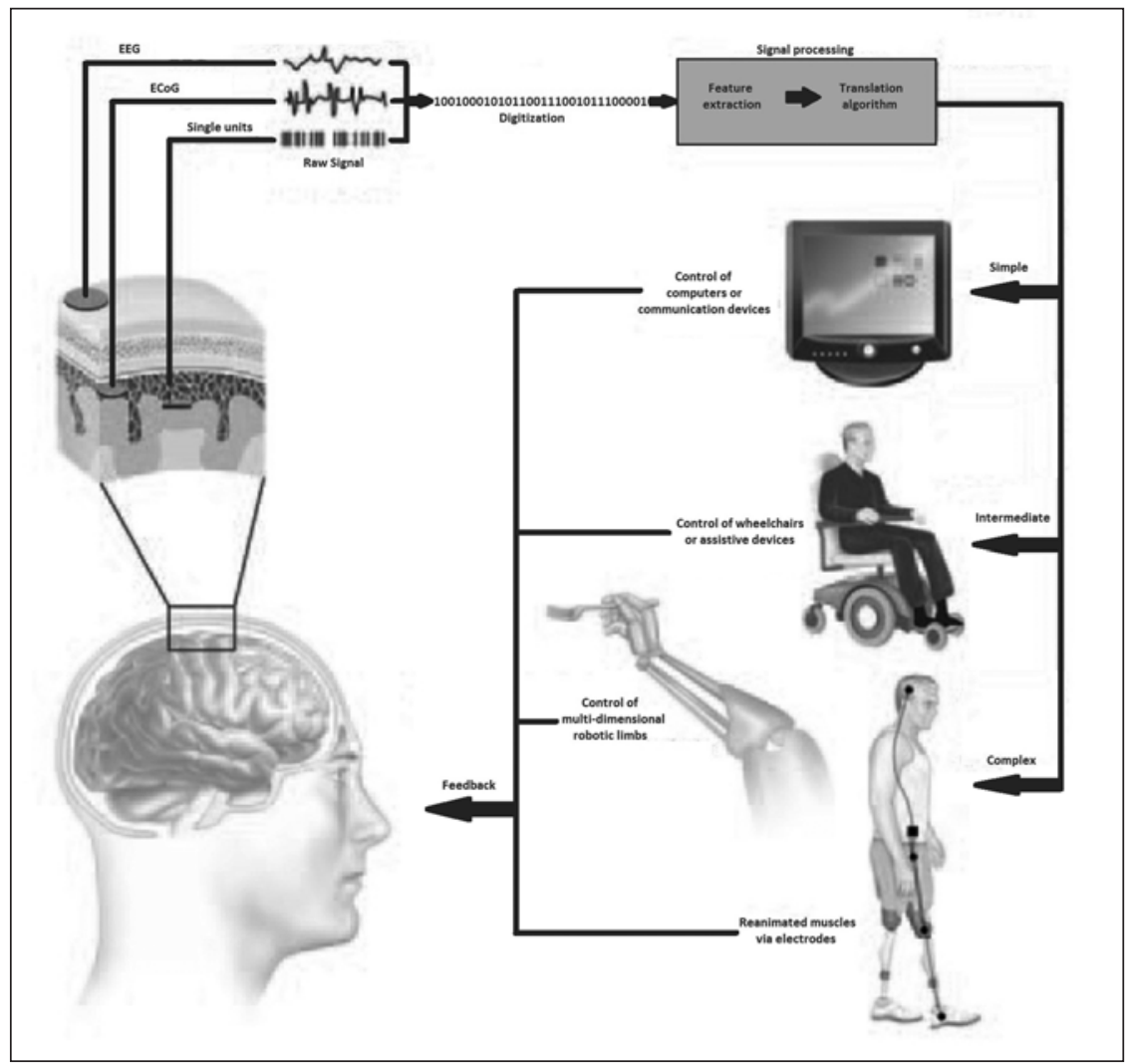

Figure 1: BMI schematic diagram showing the 3 major components: 1) signal acquisition; 2) signal processing; and 3) output execution. (reproduced from Leuthardt et al., 2006) 
neurophysiology (including neuroplasticity) remain largely unclear, underscoring just how remarkable it is that the human brain can learn to take control of devices such as BMIs. Machine learning algorithms can also use feedback to carry some of the burden of adaptation, potentially permitting BMI control to occur with reduced mental effort. ${ }^{44}$ Some have also imagined generating feedback signals by recording sensory information from cortex or peripheral nerves to facilitate more precise control of BMI systems. ${ }^{45,46}$

\section{3) Neural Signal Acquisition for BMIs}

\section{1) Noninvasive Signal Acquisition}

Noninvasive signal acquisition employs technology that can be used to measure brain activity without first requiring neurosurgical implantation. Such technology is attractive as it avoids the real and perceived risks associated with more invasive forms of BMI, but is weakened by limitations related to signal quality and portability.

\subsection{1) Scalp EEG}

Decades of EEG experiments have shown that scalp recordings can detect a number of distinct patterns of brain activity that reflect some degree of volitional control. ${ }^{47}$ Due to the distance and low-pass filtering property of the intervening skull between brain and electrode, these recordings reflect gross neural activity with about $3 \mathrm{~cm}$ spatial discrimination and are limited to the $0-35 \mathrm{~Hz}$ frequency range. ${ }^{17,48}$ The EEG signal is believed to primarily reflect synchronous neural firing and/or an accumulation of extra-cellular charge from prolonged activity or inhibition. ${ }^{49-51}$ Certain patterns, including slow cortical potentials, rolandic beta and mu rhythms (also known as sensorimotor rhythms (SMRs), and the P300 wave, have been used to train individuals to gain volitional control over components in their environment, although not all patients are successful in this process. ${ }^{52}$ This training requires significant effort and time, and BMI systems based on scalp EEG recordings have, to date, only achieved low to moderate levels of performance, with the greatest advance being moderately accurate control over a computer cursor. ${ }^{53}$ The EEG systems also have a number of limitations in that they are highly vulnerable to ambient electrical fields and contamination from muscular electrical activity, which could impair BMI performance or, at worst, cause gross malfunction.

\subsection{2) Other forms of non-invasive signal acquistion}

Other alternatives to scalp EEG have been explored in the context of acquiring non-invasive neural signals to drive BMI systems. Principally, these alternatives are based upon functional brain imaging techniques, such as magnetoencephalography (MEG), functional magnetic resonance imaging (fMRI), positron emission tomography (PET), near-infrared (NIR) imaging, and functional transcranial Doppler (fTCD) sonography.$^{54}$ While MEG and fMRI do not offer the simplicity and portability of electrical recording, their non-invasiveness and ability to represent brain-wide neural activity make them potentially attractive for stationary BMI applications like computer cursor control. Positron emission tomography is valuable only in a limited research environment, due to the need for injection of a radioactive tracer. Functional transcranial Doppler is a newer concept, detecting flow changes in intracranial arteries, but is currently limited to only the largest of vessels. ${ }^{54}$ However, near-infrared (NIR) optical imaging could potentially provide portable and accurate non-invasive recordings if certain technical challenges are resolved. ${ }^{55-57}$ Although the mechanism has yet to be fully understood, subtle changes in neuronal membranes during firing cause a difference in NIR attenuation, which could be detected using a noninvasive apparatus. ${ }^{55}$ In any case, in the context of non-invasive BMIs, it is critically important to also consider non-BMI technologies like voice and movement-controlled assistive devices, including those that detect eye movements, tongue movements, or sip and puff actions. These options are simple and low-cost, and, for many individuals, may provide for sufficient control over their environment to render BMI approaches unsuitable or impractical.

\section{2) Invasive Signal Acquisition}

Neural signals acquired from within the cranium have the potential to more faithfully reproduce desired motor output at the expense of some risk of injury to the brain. Several different modalities of intracranial electrophysiological recordings have been developed, such as electrocorticography (ECoG), single unit recordings, and local field potentials (LFPs), which can be viewed along an anatomical continuum ranging from extradural techniques to intraparenchymal methods where the blood-brain barrier is disrupted (Figure 2).

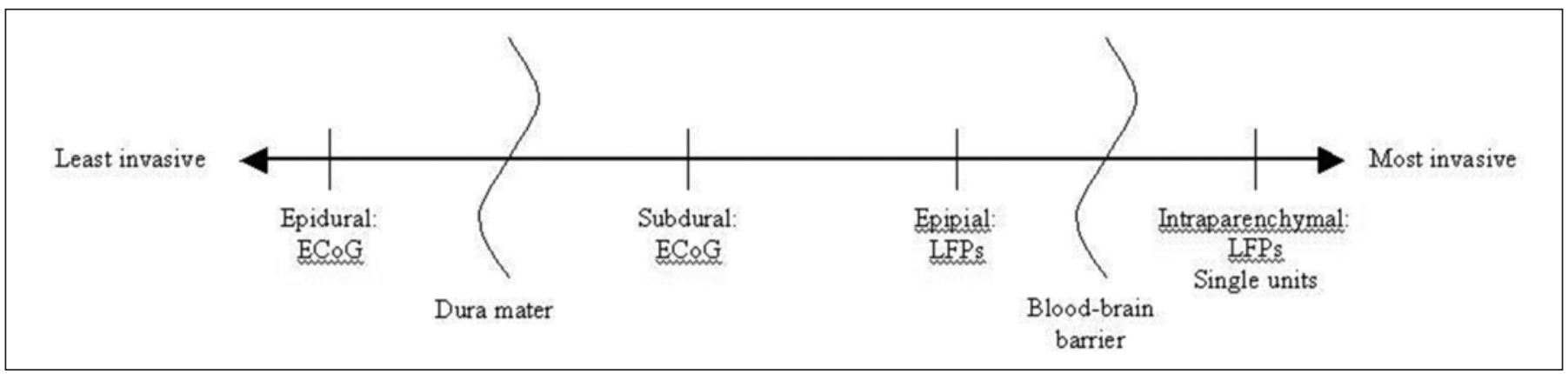

Figure 2: Continuum of invasive recording modalities. ECoG=electrocorticography; LFPs=local field potentials 


\subsection{1) Electrocorticography $(\mathrm{ECo} G)$}

Electrocorticography is the least invasive of the invasive signal acquisition methods. Almost invariably, ECoG signals are obtained by epidural or subdural electrode strips or grids implanted through burr holes or craniotomy, although one report described the use of conductive skull screws. ${ }^{58}$ These epidural or subdural electrodes have been employed in humans for such tasks as identification of seizure foci, treatment of chronic pain, and functional mapping of the cortex. ${ }^{17,59-62}$ Subdural ECoG electrodes are believed to be safer and longer lasting than those placed directly in brain parenchyma. ${ }^{63}$ Further, one report found epidural and subdural ECoG signals to be nearly equivalent, suggesting that opening the dura may not be necessary. ${ }^{64}$ While similar to EEG and generated by the same neuronal sources, ECoG's superior signal quality gives it comparatively five times the amplitude, a wider bandwidth encompassing frequencies from $0-200 \mathrm{~Hz}$, and better spatial resolution on the order of nearly $1 \mathrm{~mm} .{ }^{17}$ Perhaps the greatest advantage is the ability to detect gamma frequency oscillations $(>30 \mathrm{~Hz})$, which are believed to reflect synchronous firing in intracortical circuits comprised of a small number of neurons confined to a few $\mathrm{mm}^{2}$ of cortex. ${ }^{60,65-68}$ Practical successes with ECoG in human BMI research have included 3-dimensional control of a computer cursor (two directions and a trigger) and differentiating between four different speech phonemes. ${ }^{62,69,70}$ However, there has yet to be a clinical trial of ECoG purely for BMI purposes, as current studies have only been in patients with arrays placed for other reasons. It also remains to be seen if $\mathrm{ECoG}$ provides a reliable signal in the context of head motion, as the movement of the brain relative to electrodes could reduce effectiveness, particularly in patients with atrophy or pre-existing cortical injury.

\subsection{2) Single Unit, Multi-Unit, and Neuronal Ensemble Recordings}

The spiking activity of individual neurons produces 100-300 $\mu \mathrm{v}$ signals that can be recorded by intraparenchymal electrodes where the recording surface is within about $100 \mu \mathrm{m} .{ }^{23}$ Such recordings are termed 'single-units' for individual neurons, or 'multi-units' for the superimposed spiking activity of several neurons. Electrodes used to acquire single or multi-unit recordings are typically inserted using stereotactic - and usually frame-based-neurosurgical techniques.

Pioneering experiments by Fetz and Schmidt showed monkeys could gain fine control via operant conditioning over the firing rates of individual M1 neurons. ${ }^{32,40}$ This principle led to the development of glass cone electrodes coated with neurotrophic factors that Kennedy and Bakay implanted in five paralyzed humans, obtaining recordings over several years that have been used to control several simple devices. ${ }^{4,42,71}$ However, only minimal data from these experiments has been published, and the methods have not been widely pursued by other research groups.

At the same time, tremendous progress has been made in the area of neuronal ensemble recordings, which are simultaneous recordings of many neurons over multiple channels. Neuronal ensembles represent the logical evolution of single and multiunit recordings, and have favourable properties that lend themselves to BMI systems. Georgopoulos demonstrated that many M1 neurons have a preferred direction for movement in which they fire maximally, and subsequent breakthroughs by other groups showed that an ensemble comprised of a modest number of neurons could accurately predict the $2 \mathrm{D}$ or $3 \mathrm{D}$ direction of movement. ${ }^{32,43,72,73}$ Subsequently, specialized recording arrays have been developed with up to 100 microwire or silicone probes that penetrate the cortex and record on multiple channels. ${ }^{23,74}$ Several groups have demonstrated that animals with these implanted arrays can achieve continuous 3D control over robotic devices, including Nicolelis' group that decoded detailed kinematic information in real-time while recording from over 300 neurons. ${ }^{20,27,75-78}$ Schwartz's group released a video showing monkeys casually feeding themselves via a $4 \mathrm{D}$ robotic arm (3D movement plus a gripper), while able to chew and look around, controlled by more than 100 single unit recordings. ${ }^{79}$ In 2006, human trials began with Donoghue's group implanting four paralyzed individuals with 96-electrode 'Braingate' arrays, who subsequently gained up to 3D control over a number of devices. ${ }^{15,80}$ However, in spite of the accomplishments made using ensemble recordings, numerous technical challenges remain. Of primary concern is the gliotic tissue response that encapsulates electrodes and degrades the quality of recordings, consistently rendering them unusable in months or a few years. ${ }^{80-84}$ Also, the signals are highly variable from day to day due to micromotion and are susceptible to sharp head movements, often making it difficult to know if the same neurons are being recorded. ${ }^{45}$ Efforts have been made to accommodate this motion with microelectromechanical systems (MEMS) that can move electrodes on the micrometer $(\mu \mathrm{m})$ scale and track target neurons. ${ }^{85}$ Other difficulties are insulation breakdown, amplifying the electrical signals while minimizing noise, relaying the signals out of the skull wirelessly, and powering the system without generating excess heat. ${ }^{6,86-88}$ These obstacles must be overcome to allow chronic durable recordings that can be reliably and effectively used within a BMI system.

\subsection{3) Local Field Potentials}

Local Field Potentials (LFPs) are also recorded from intraparenchymal microelectrodes, being most often used in scenarios where individual action potentials cannot be identified..$^{23,89,90}$ These recordings have been studied in humans in several clinical settings, such as in the context of using depth electrodes for identifying epileptic foci or during deep brain stimulation (DBS) electrode placement..$^{90}$ Historically, LFPs have been relatively overlooked in favour of neuronal spiking activity, possibly due to the difficulty in interpreting LFPs. ${ }^{91}$ This trend is rapidly changing, however, as recent advances have shown that local field potentials are precise measures of neural activity, sometimes more robust than data generated by single units. ${ }^{92-96}$ The current view is that the LFP signal reflects synaptic potentials averaged across a local region of about $250 \mu \mathrm{m} .{ }^{97}$ Thus, the activity of small numbers of neurons is captured, including both the average activity level and oscillations due to synchronous firing. Oscillations tend to be primarily in the gamma range, suggesting that LFP and ECoG recordings overlap in their detection of neural activity, but with LFPs able to accurately record the high-gamma (100-400 Hz) range. ${ }^{62,94,98}$ Recent work in fact suggests that high-gamma LFP recordings may yield even more movement information. ${ }^{96}$ 
Interestingly, Kellis et al demonstrated that it is also possible to capture an LFP signal less invasively from the pial surface, with better spatial resolution than is possible with subdural ECoG grids. ${ }^{99}$

\section{3) Anatomic Target Regions for Neural Signals in BMIs}

The complexity of motor planning and execution in the brain naturally implies existence of a number of candidate brain regions from which data can be acquired to drive a BMI system. Choosing between these anatomic locales, and-as we have already seen-using the appropriate recording modality to characterize the neural activity in each, is critically important in the design of any BMI. Several regions of interest relevant to BMIs are listed in Table 1.

To date, the majority of invasive recordings have focused on the primary motor cortex (M1). ${ }^{27}$ Individual M1 neurons have been shown to fire in relation to a number of different movement parameters, including direction, velocity, acceleration, and force. . $^{27,32,73,100,101}$ Nearby neurons, within about $200 \mu \mathrm{m}$, tend to encode the same parameter suggesting that there may be no additional benefit to recording on a smaller scale. ${ }^{101}$ Further, some debate continues over the question of whether M1 ultimately encodes the motor activity of individual muscles or, instead, more complex overall movements. ${ }^{28,100,102}$ Recent tractography work in macaques showed that M1 neurons making direct synapses with spinal motoneurons were entirely confined to the caudal M1 buried in the central sulcus. ${ }^{103}$ Accordingly, researchers who have placed recording electrode arrays have found that M1 neurons primarily encode kinematics rather than muscles, since recording arrays are almost invariably placed on the precentral gyrus. ${ }^{104-106}$ Most designers of BMI systems have typically based their systems on neural information thought to encode for overall kinematic intent, but it is conceivable that complex output devices like exoskeletons could be controlled in many dimensions if intended outputs to individual effector muscles could be simultaneously harnessed.

Several other brain regions involved in movement have also been considered. High-level planning areas such as the posterior parietal cortex (PPC) and the prefrontal cortex (PFC) have the advantage that they appear to encode abstract movement goals in advance of the actual movement. ${ }^{107-111}$ Andersen's group at Caltech has termed BMIs using these signals "cognitive-based" and demonstrated that they require small numbers of neurons and can predict movement goals in less time than it takes to decode kinematic information. ${ }^{72,89,107,109,110}$ The PFC may be particularly advantageous because it responds readily to conscious control, which could help mediate the magnitude and duration of machine-generated movements. ${ }^{112}$ Movement preparation areas such as the premotor (PM) cortex and supplementary motor area (SMA) have also been extensively studied and have similarly been found to have favourable properties. ${ }^{78,89,113-118}$ The ventral PM cortex (PMv) is highly active in spatially-guided movement whereas the dorsal region (PMd) appears to be involved in arbitrary rule-based movement. ${ }^{78,89,114-117}$ The SMA may be useful due to its association with the planning of movements, particularly those that are internal or memory-guided. ${ }^{113,118,119}$ The rostral SMA shows early activation whereas the caudal SMA is more active during movement itself and is highly correlated with movement kinematics. ${ }^{118,120}$

It has been widely established that the basal ganglia are critical to motor preparation and execution. ${ }^{121,122}$ Invasive recordings in humans during DBS electrode placement have shown that both the subthalamic nucleus (STN) and the ventrolateral (VL) thalamic nucleus are active before and during different movement types. ${ }^{121-123}$ One group has investigated these targets in DBS patients with a focus on the execution phase of movement, obtaining 32-electrode recordings with a specialized microwire array. ${ }^{124}$ This work demonstrated that both

Table 1: Summary of common anatomical targets for BMIs

\begin{tabular}{|c|c|c|c|}
\hline \multirow{5}{*}{$\begin{array}{l}\text { Stage } \\
\text { Movement } \\
\text { execution }\end{array}$} & \multicolumn{2}{|c|}{ Anatomical Target } & Function \\
\hline & Motor cortex (M1) & Precentral gyrus & $\begin{array}{l}\text { Controls motor output; cells fire in relation to velocity, } \\
\text { direction, acceleration, and force }\end{array}$ \\
\hline & & $\begin{array}{l}\text { Central sulcus (motor } \\
\text { bank) }\end{array}$ & Output area of M1 \\
\hline & Motor thalamus & & $\begin{array}{c}\text { Monitor of motor output receiving projections from layer V } \\
\text { M1 cells, basal ganglia, and cerebellum }\end{array}$ \\
\hline & Basal ganglia & $\begin{array}{l}\text { Subthalamic nucleus } \\
\text { (STN) }\end{array}$ & $\begin{array}{c}\text { Somatotopically organized, active throughout movement, } \\
\text { firing rates related to force; also involved in movement } \\
\text { preparation }\end{array}$ \\
\hline \multirow[t]{5}{*}{$\begin{array}{l}\text { Movement } \\
\text { preparation }\end{array}$} & $\begin{array}{l}\text { Premotor cortex } \\
\text { (PM) }\end{array}$ & Ventral (PMv) & Spatially guided movement \\
\hline & & Dorsal (PMd) & Arbitrary rule-based movement \\
\hline & Supplementary & Pre-SMA & Movement planning \\
\hline & Motor Area (SMA) & (rostral SMA) & \\
\hline & & $\begin{array}{l}\text { SMA proper } \\
\text { (caudal SMA) }\end{array}$ & Movement kinematics, active during execution \\
\hline \multirow[t]{2}{*}{$\begin{array}{l}\text { Movement } \\
\text { planning }\end{array}$} & $\begin{array}{l}\text { Posterior parietal } \\
\text { cortex (PPC) }\end{array}$ & & $\begin{array}{l}\text { Integration of visual and sensory information, transformation } \\
\text { into common reference frame for motor planning }\end{array}$ \\
\hline & $\begin{array}{l}\text { Prefrontal cortex } \\
\text { (PFC) }\end{array}$ & & $\begin{array}{c}\text { Goal-directed behaviour and spatial processing, } \\
\text { consciously control }\end{array}$ \\
\hline
\end{tabular}


the STN and VL nucleus are highly active during gripping, and small numbers of neurons could accurately predict grip force. These results provide proof of concept that subcortical recordings are feasible and that they should also merit consideration as potential BMI signals, with the caveat that electrode insertion trauma must be minimized to avoid inadvertent lesioning effects.

Though not a brain structure per se, the spinal cord may also be a signal source, both at levels above and below a transection, if reliable signals can be obtained. Above the transection, recording may be done from descending motor tracts, harnessing detailed movement instructions or locomotive commands. However, recording from white-matter tracts is a substantial challenge, as discrimination between signals carried on densely packed axons exceeds the capabilities of current methods. Proposed but as yet unproven techniques include electrical, magnetic, and optical recordings. ${ }^{125,126}$ Below the transection, recordings from dorsal root ganglia or dorsal horns could provide invaluable feedback information for FES reanimation of paralyzed muscles. Systems have been proposed that could restore bipedal locomotion after spinal cord injury (SCI) using electronic central pattern generators (CPGs) that perform microstimulation of ventral horns, guided by appropriate sensory feedback. ${ }^{127}$ Proof of this concept has been demonstrated in animal models, restoring four-legged locomotion after complete spinal cord transection using CPG circuitry that closely replicates neuronal function, known as 'neuromorphic' technology. ${ }^{128}$

Ultimately, the creation of an optimally functional BMI may require input signals from multiple anatomic sources. Some have suggested a BMI system recording from thousands of individual neurons across cortical, subcortical, and peripheral structures. ${ }^{21}$ Others contend that less invasive methods like ECoG can extract sufficient information while monitoring a wider territory of cortex..$^{10}$ Regardless of recording modality, the selection of suitable anatomic targets will depend on the ability to decode precise function in each brain region.

\section{4) Establishing Clinical Utility of BMIs}

\section{1) Patient Populations Likely to Benefit from BMIs}

Patients with mild to moderate disabilities can be defined as retaining complex motor function in at least some parts of the body, such as hand movement or voice control. Such conditions often include SCI, amputation, unilateral stroke, Parkinson Disease (PD), or other movement disorders. While these individuals are often profoundly affected by their deficits, optimal treatment tends to focus on maximizing the use of intact capabilities rather than trying to replace lost function. Technologies such as voice and movement-based assistive devices are likely to provide significant benefit for this group with minimal risk and cost, as are other human-machine interfaces that do not involve the central nervous system. For example, electromyelogram-based prostheses in amputees can take advantage of the fine control permitted by an intact motor pathway. ${ }^{38,129}$ More aggressive approaches like implanted BMIs will only be considered in the mild-moderately disabled population when these technologies mature sufficiently to prove they are safe, durable, and provide significant gains in function over the alternatives. An exception to this may be tetraplegic patients, who retain no significant motor function other than voice and facial expression. Some in this population may place sufficient value on regaining functions like hand and arm movements or basic mobility to justify a neurosurgical procedure. $^{130}$

Patients with complete or near-complete motor deficits are those who do not retain any complex motor functions like voice or hand movements. Such individuals may be in a locked-in state (LIS) where expressive communication is severely limited, but they do retain rudimentary control of at least one muscle, or they may lose all motor output and enter the completely locked-in state (CLIS). ${ }^{131}$ Conditions that can cause this level of disability include ALS, subcortical stroke, Guillain-Barre syndrome, rare cases of parkinson's disease, and advanced multiple sclerosis (MS). ${ }^{131}$ While some of these conditions also involve cognitive deficits, many of these patients retain significant cognitive abilities and therefore have the potential to obtain substantial benefit from a BMI. However, it is important to discuss noninvasive options with these patients in a balanced and honest manner, given the possibility that their desperate clinical situation may push them to select more aggressive and experimental treatment alternatives. From experience with EEGbased BMIs, it is known that training LIS or CLIS patients is often highly demanding, and so it is crucial to consider other factors, such as the patient's motivation, attitude, social circumstances and caregiver support, that could influence the eventual outcome with an implanted BMI. ${ }^{132}$ Ultimately, if the patient's goals involve regaining significant function and their cognition is intact such that they can evaluate and accept the risks associated with implantation, then these patients are well suited to be candidates for an implanted BMI. In these cases, a BMI with the absolutely lowest cognitive demand (see below) is ideal, as the primary goal is to provide a novel output channel for communication. This might take a form such as a single unit or LFP-based BMI recording only from the hand area of M1, and controlling a computer mouse with three DOFs (2D movement plus click).

\section{2) Current Status of Clinical BMIs}

At present, clinical data regarding the use of BMIs in humans is limited, due to the experimental status of many of these devices. The earliest reports of human clinical trials have primarily involved patients with ALS and other conditions causing near-complete motor disability.

\subsection{1) $A L S$}

The majority of BMI applications targeted to ALS patients have utilized EEG as the signal source. In 1999 and 2003, Birbaumer et al reported results of a spelling device used by nine ALS patients and two with other conditions who were nearly locked-in or completely locked-in. ${ }^{5}$ This system was based on gaining volitional control of slow cortical potentials (SCPs) and all seven patients who were not completely locked-in achieved moderate accuracy $(>75 \%)$, but their performance averaged only one character per second and they required hundreds of short training sessions. All four patients who were completely lockedin were unable to gain control of the device, for reasons that are unknown. Other EEG devices have also been tested clinically, 
including several trials using P300 event-related potentials (ERPs). In 2006, Sellers and Donchin demonstrated that three ALS patients could utilize a P300-based system with similar results to able-bodied persons, although the subjects' motor disabilities were only moderate at the time of the trial. ${ }^{9}$ Subsequently, Nijboer et al showed that six of eight ALS patients with moderate to severe deficits could gain control and produce 1-4 characters per minute with up to $92 \%$ accuracy. ${ }^{8}$ Other work has focused on harnessing SMRs, including one trial demonstrating that four severely disabled ALS patients could all gain nearly $80 \%$ accuracy in a binary selection task. ${ }^{7}$ Overall, the various EEG methodologies using SCPs, P300 ERPs, and SMRs have all demonstrated roughly equivalent performance and moderate utility as a communication channel for near locked-in individuals, but in one direct comparison study in seven ALS patients, SMRs appeared to have the distinct advantage of reduced training time. ${ }^{131}$

Implanted BMIs have also been targeted to ALS, with early trials showing some promising preliminary results. Among five patients implanted with neurotrophic glass cone electrodes within M1 or Broca's area, two had ALS, while two others had brainstem strokes and one had mitochondrial myopathy. $4,42,71$ Unfortunately, after gaining binary control with minimal training, the first ALS patient died two months after implantation due to her disease, while the second ALS patient only completed two sessions of recordings. ${ }^{4}$ In the initial BrainGate trials for tetraplegia performed by Donoghue's group, one ALS patient was recruited, along with two that had upper-SCI and one with a brainstem stroke. ${ }^{133}$ All of these patients were able to gain nearimmediate mastery of several output devices, with a point-andclick computer cursor being of particular value. However, the results from the BrainGate pilot study are only one to two years after implantation, and show a progressive decline in number of viable signals recorded. ${ }^{6}$ It may be several years until longerterm data surface; these data are likely to come from the BrainGate2 trial which began the process of recruiting approximately 15 tetraplegic patients in $2010 .{ }^{134}$

\subsection{2) SCI, Brainstem Stroke}

In non-progressive causes of tetraplegia, like SCI and brainstem stroke, most patients retain cranial nerve function that enables speech and facial movements. Thus, few attempts have been made to apply EEG-based BMIs to this population. One exception is a report from Pfurtscheller et al in 2003 that described an incomplete tetraplegic patient (retaining left shoulder extension) who regained hand-grasp control using EEG-recorded SMRs. ${ }^{39}$ Although simplistic in design, this was among the earliest human applications of FES to reanimate a paralyzed limb, and served as an intriguing proof-of-concept. Nevertheless, to date, EEG-based BMIs do not seem to provide sufficient performance to benefit tetraplegics with complete limb paralysis.

In contrast, several patients with complete tetraplegia due to SCI and brainstem stroke have been tested with implanted BMI prototypes. The two patients with brainstem strokes that were implanted with glass cone electrodes both achieved several years of stable recordings, and one was able to control devices such as a computer cursor and a 'cyber hand' while the other produced seven different speech phonemes. ${ }^{4}$ Among the BrainGate participants, 96-channel multielectrode recordings of the arm area of M1 revealed that significant motor cortex function is preserved for years after sustaining a complete SCI or brainstem stroke ${ }^{6,80}$ Further, the patients with these injuries could quickly gain control over various output devices solely using motor imagery, as they had not performed physical movements for several years. However, for the tetraplegics enrolled in the BrainGate trial, it may have been disappointing that they were not able to match the results seen in animal trials and gain masterful control over devices like 3D or 4D robotic arms. As the technology matures, high performance BMI systems will have to significantly outperform voice-controlled devices to be useful in the tetraplegic population, as voice-controlled devices like wheelchairs have already entered the commercial market. ${ }^{135}$

\subsection{3) Unilateral Stroke}

In contrast to the relatively small group of individuals afflicted with near-complete paralysis, the staggering number of people living with stroke-induced hemiparesis is an order of magnitude greater. ${ }^{136}$ In particular, chronic hand plegia is a common result, with no effective treatments currently available. ${ }^{137}$ Few reports have discussed serious BMI efforts targeted to this group, although one demonstrated that a simple EEG-driven hand orthosis successfully restored hand grasp in six of eight stroke patients with only modest training. ${ }^{138}$ A study by Wisneski et al demonstrated that ipsilateral motor cortex activity detected with ECoG could also be used to accurately predict hand movements, although this work was performed in able-bodied epilepsy patients. ${ }^{139}$ If ipsilateral motor signals can be robustly decoded and differentiated from more potent contralateral signals, the potential targets for BMI applications will grow many-fold. ${ }^{18}$

\section{5) Criteria for Evaluating BMIs}

As implantable BMI technologies evolve and become clinically available, it is imperative that clinicians are equipped to evaluate and compare these products with standard alternatives. To this end, a risk-benefit analysis of implanted BMIs should include the following criteria: safety, performance, durability, ease-of-use, cost, and individual suitability (Table 2).

Safety is of utmost concern when considering the elective intracranial implantation of a novel device. Reasonable estimates of surgical risk can be obtained from the established neurosurgical literature. For example, it would be expected that the risks associated with subdural ECoG implantation would closely parallel the reported risks for subdural strip electrode placement, and would include such complications as wound infections (up to 2.5\%), meningitis (up to 2.5\%), and osteomyelitis (up to $0.8 \%$ ), as well as non-infectious complications like cerebrospinal fluid leak, and subdural hemorrhage. ${ }^{26,81,140,141}$ For devices implanted directly into the parenchyma to obtain LFPs or single unit recordings, risks of deep hemorrhage and accompanying permanent neurological injury must be expected. Based on the DBS literature, the overall risk of permanent injury in DBS electrode placement has been reported as less than $1 \% .{ }^{142}$ Unfortunately, the risks associated with multielectrode microarray placement are not wellcharacterized and appear to be dependent both on specific device used and the method of placement. ${ }^{81}$ 
Table 2: BMI comparison table

\begin{tabular}{|c|c|c|c|c|c|c|c|}
\hline \multirow{2}{*}{\multicolumn{2}{|c|}{$\begin{array}{l}\text { Signal Type } \\
\text { ECoG }\end{array}$}} & Safety & Performance & Durability & Ease-of-use & Cost & Individual Suitability \\
\hline & & $\begin{array}{ll}\text { - } & \text { Moderately } \\
& \text { invasive } \\
\text { - } & \text { Likely reliable }\end{array}$ & $\begin{array}{ll}\text { - } & \text { DOF: } 3 \\
\text { - } & \text { Bandwidth: medium } \\
\text { - } & \text { Aerritory: broad } \\
\text { - } & \text { Delay: moderate } \\
\end{array}$ & Medium-high & $\begin{array}{ll}\text { - } & \text { Portable } \\
\text { - } & \text { Parameters likely stable } \\
& \text { Operating systems not well } \\
\text { - } & \text { Cognitished } \\
\end{array}$ & High & - $\quad$ Need to accept surgical risk \\
\hline \multicolumn{2}{|l|}{ LFPs } & $\begin{array}{ll}\text { - } & \text { Highly } \\
& \text { invasive } \\
\text { - } & \text { Likely reliable }\end{array}$ & $\begin{array}{ll}\text { - } & \text { DOF: } 3 \\
\text { - } & \text { Bandwidth: high } \\
\text { - } & \text { Acritory: narrow } \\
\text {. } & \text { Delay: moderate } \\
\end{array}$ & Medium & $\begin{array}{ll}\text { - } & \text { Portable } \\
\text { - } & \text { Parameters likely stable } \\
\text { - } & \text { Operating systems not well } \\
& \text { established } \\
\text { - } & \text { Cognitive demand: low } \\
\end{array}$ & High & $\begin{array}{l}\text { - } \quad \text { Desire for high performance } \\
\text { - }\end{array}$ \\
\hline \multicolumn{2}{|c|}{ Single/multi-units } & $\begin{array}{ll}\text { - } & \text { Highly } \\
& \text { invasive } \\
\text { - } & \text { Unreliable }\end{array}$ & $\begin{array}{ll}\text { - } & \text { DOF: } 4 \\
\text { - } & \text { Bandwidth: high }(6.5 \mathrm{bits} / \mathrm{s}) \\
\dot{-} & \text { Aerritory: narrow } \\
\text { - } & \text { Delay: moderate }(150 \mathrm{~ms}) \\
\end{array}$ & Low & $\begin{array}{ll}\text { - } & \text { Portable } \\
\text { - } & \text { Parameters unstable } \\
& \text { Operating systems fairly well } \\
& \text { established } \\
\text { - } & \text { Cognitive demand: low } \\
\end{array}$ & High & $\begin{array}{l}\text { - } \quad \text { Desire for high performance } \\
\text { - }\end{array}$ \\
\hline \multicolumn{2}{|l|}{ EEG } & $\begin{array}{ll}\text { - } & \text { Non-invasive } \\
\text { Inaccurate and } \\
\text { unreliable }\end{array}$ & $\begin{array}{ll}\text { - } & \text { DOF: } 3 \text { (2+trigger) } \\
\text { - } & \text { Bandwidth: low }(0.5 \text { bits/s) } \\
\text {. } & \text { Territory: broad } \\
\text { - } & \text { Decuracy: low-medium } \\
\end{array}$ & High & $\begin{array}{ll}\dot{*} & \text { Portable } \\
\dot{*} & \text { Parameters somewhat unstable } \\
\text { - } & \text { Cognitive demand: high }\end{array}$ & Medium & $\begin{array}{l}\text { - Well-suited for low-performance } \\
\text { tasks like communication } \\
\text { - } \quad \text { Unsafe for wheelchair control } \\
\text { Need to tolerate external apparatus }\end{array}$ \\
\hline \multirow[t]{5}{*}{ Alternatives } & MEG & $\begin{array}{l}\text { Non-invasive } \\
\quad \text { Unreliable }\end{array}$ & $\begin{array}{ll}\text { - } & \text { DOF: } 2 \\
\text { - } & \text { Bandwidth: low-medium } \\
\text { - } & \text { Territory: broad } \\
\text { - } & \text { Accuracy: unknown } \\
& \text { Delay: moderate-high } \\
\end{array}$ & High & $\begin{array}{ll}\text { - } & \text { Unlikely to be portable } \\
\text { - } & \text { Parameters unstable } \\
\text { - } & \text { Cogniting systems not established } \\
& \text { demand: likely high }\end{array}$ & High & - Need to tolerate external apparatus \\
\hline & FMRI & $\begin{array}{ll}\text { - } & \text { Non-invasive } \\
\text { - } & \text { Likely reliable }\end{array}$ & $\begin{array}{ll}\cdot & \text { Performance: likely poor } \\
\text { - } & \text { Territory: broad } \\
\text { - } & \text { Decuracy: high } \\
\end{array}$ & High & $\begin{array}{ll}\cdot & \text { Non-portable } \\
\dot{*} & \text { Parameters likely stable } \\
\text { - } & \text { Operating systems not established } \\
\end{array}$ & Very high & - Need to tolerate external apparatus \\
\hline & Optical (NIR) & $\begin{array}{ll}\text { Non-invasive } \\
\text { Likely } \\
\text { unreliable }\end{array}$ & $\begin{array}{ll}\text { - } & \text { Performance: high (if fast } \\
& \text { signal detectable) } \\
\text { - } & \text { Territory: broad } \\
\text { - } & \text { Accuracy: unknown } \\
\text { - } & \text { Delay: low (fast signal, slow } \\
& \text { signal has long delay) } \\
\end{array}$ & Likely high & $\begin{array}{ll} & \text { Portable } \\
\dot{*} & \text { Parameters likely stable } \\
\text { - } & \text { Operating systems not established } \\
& \text { Cognitive demand: low }\end{array}$ & High & - $\quad$ Need to tolerate external apparatus \\
\hline & $\begin{array}{l}\text { Movement } \\
\text { (eye/ } \\
\text { tongue/ EMG) }\end{array}$ & $\begin{array}{ll} & \begin{array}{l}\text { Non-invasive } \\
\text { (or minimal }\end{array} \\
\text { for EMG) } \\
\text { Reliable }\end{array}$ & $\begin{array}{ll} & \text { DOF: likely 2 (depends on } \\
\text { motor capabilities) } \\
\text {. } & \text { Bandwidth: medium } \\
\text {. } & \text { Accuracy: high } \\
\text { Delay: low }\end{array}$ & High & $\begin{array}{ll}\cdot & \text { Portable } \\
\text { - } & \text { Parameters stable } \\
\text { - } & \text { Operating systems established } \\
\text { - } & \text { Cognitive demand: low-medium }\end{array}$ & Low & - $\quad$ Requires some intact motor output \\
\hline & Voice & $\begin{array}{ll}\text { Non-invasive } \\
\quad \text { Reliable }\end{array}$ & $\begin{array}{ll}\cdot & \text { DOF: many } \\
\cdot & \text { Bandwidth: medium } \\
\cdot & \text { Accuracy: medium-high } \\
\cdot & \text { Delay: medium-high }\end{array}$ & High & $\begin{array}{ll} & \text { Portable } \\
\text { - } & \text { Parameters stable } \\
\text { - } & \text { Operating systems established } \\
\text { Cognitive demand: low-medium }\end{array}$ & Low & - $\quad$ Requires intact speech \\
\hline
\end{tabular}

Other safety concerns include hazards like radiation, heat, immunological responses, battery leaks, or mechanical damage to neural tissue with sudden acceleration or deceleration. Many of these issues are dependent on the specific device in use, and must be rigorously characterized in animal models and early human trials so that surgeons and patients can properly weigh these risks. Unreliable performance leading to gross malfunction is a particularly troubling concern, which may be more likely to occur in EEG-based BMIs due to electrical noise or in single unit systems with sudden head motion. Failsafe mechanisms are essential so that BMI systems can be quickly shut down or reset in emergency scenarios.

The quantification of performance in BMIs can be considered in terms of several variables including degrees of freedom (DOFs), bandwidth, accuracy, and delay. Degrees of freedom are the number of independent variables or directions that can be controlled simultaneously. For example, the shoulder has three DOFs (flexion-extension, abduction-adduction, external-internal rotation) whereas the elbow has only one DOF (flexionextension). The maximum number of DOFs possible in a BMI depends on such issues as the signal quality, its correlation with movement, the neural territory covered, and the number of independent components extracted from the signal. As an example, single unit recordings and LFPs have an advantage in signal quality, but ECoG is able to cover wider cortical territory. Bandwidth is the information transfer rate, usually measured in bits per second (bits/s). In a system with only one DOF, bandwidth might reflect the speed of control over an open-close gripper. Accuracy reflects both the error rate of incorrect actions and the rate of false positive actions in systems where a rest state exists. Local field potential and single unit BMIs have demonstrated highest accuracy thus far, but dedicated ECoG BMI research could potentially negate this advantage. $5,53,79,80,92-$ 95,143,144 Delay is the time lag between an intended action and its actual execution. Most BMI systems show at least a moderate delay due to the requisite computationally-intensive signal analysis. Single units may have a minor advantage in this regard, as the associated signal analysis may be more straightforward than that needed in LFPs or ECoG. Overall, the highest performing BMI systems seem likely to be based on single unit or LFP recordings, although it is premature to conclude this with any certainty.

Durability is the measure of how long BMI devices are likely to last in real-life use. Intraparenchymal BMIs are at a major 
disadvantage, as they occupy an environment where microelectrodes are quickly encapsulated in gliotic sheaths ${ }^{81-84}$ These electrodes have to record very low voltages and are highly sensitive to impedance changes, with the robustness of single unit recordings being more tenuous than LFPs. Furthermore, the intraparenchymal recordings are least amenable to replacement or repair, due to the risks associated with reoperation and stereotactic reimplantation. Experience with ECoG in epilepsy and chronic pain applications indicates that it would provide more durable recordings over time. ${ }^{63}$

Ease-of-use is a term describing how easily a user interacts with a system. The ease-of-use of a BMI can be described in terms such as portability, stability of operating parameters, and the cognitive demand required for use. The portability of any implanted BMI is likely to be superior to a non-invasive BMI, as these systems will have completely internalized wires and batteries. However, the high power requirements of multichannel single unit recordings will require larger, more powerful batteries that could be somewhat cumbersome or annoying. Operating parameters are training dependent variables such as a set of coefficients to map single unit firing rates to specific movement directions. If these operating parameters become inaccurate over time then they need to be reprogrammed with new training data, possibly in a laboratory setting. Parameters for single unit recordings are known to be unstable, which could require regular clinic visits to maintain performance. Cognitive demand is another important element to consider, as users may stay connected to BMI devices for long or indefinite stretches of time. The cognitive requirements are lowest in systems where the output action matches the imagined thought pattern, like imagining wrist movements to control a computer mouse. Electroencephalogram systems are notoriously demanding and often require long training periods to achieve mastery of control. This is less likely to be an issue with BMIs based on single unit recordings or LFPs with fine spatial resolution, but could be a moderate issue for ECoG. ${ }^{10,52}$

Cost will become a significant issue as BMIs mature and multiple alternatives become clinically available. All implanted BMIs will obviously incur a significant up-front cost due to the surgery and specialized hardware required. But it is likely that there will be differences in the frequency and nature of clinical follow-up required for patients with these systems.

Another factor in comparing BMI systems is the suitability to certain individuals or groups, driven by their limitations and the functions that are most important for them to regain. For example, one study surveyed spinal cord injury (SCI) patients and found that paraplegics rated sexual function as most important, whereas tetraplegics rated hand and arm function as their top priority. ${ }^{145}$ Patients who place higher value on regaining more complex functions may choose higher performance BMI systems, even if it means accepting additional surgical risk. However, those that only desire a communication means may find $\mathrm{ECOG}$ or a non-invasive EEG system quite adequate. In patients that have acquired cognitive deficits, cognitive demand is likely to be the most important selection factor, so an implanted BMI that minimizes this effort could be ideal.

\section{Conclusions}

In the near future, the use of implanted BMIs will likely be limited to the severely or completely disabled for whom small functional improvements would translate into substantial changes in quality of life. However, incremental improvements in numerous areas of BMI technology are occurring, with the promise of overcoming many current limitations, and a subsequent explosion of clinical applications. The future of BMI technology promises to be an exciting one. We are approaching a time when patients with BMI devices will necessarily be assessed and followed by neurologists, neurosurgeons, and other clinical practitioners.

\section{REFERENCES}

1. Drummond K. Wired.com [Internet]. Wired: Danger Room. Pentagon preps soldier telepathy push. May 14, 2009. [cited 2009 Sep 11]. Available from: http://www.wired.com/danger room/2009/05/pentagon-preps-soldier-telepathy-push/

2. Kotchetkov IS, Hwang BY, Appelboom G, Kellner CP, Connolly ES. Brain-computer interfaces: military, neurosurgical, and ethical perspective. Neurosurg Focus. 2010;28(5):E25.

3. United States Department of Defense [Internet]. Contracts. [cited 2010 Aug 8]. Available from: http://www.defenselink.mil/ contracts/contract.aspx? contractid $=2296$

4. Bartels J, Andreasen D, Ehirim P, et al. Neurotrophic electrode: method of assembly and implantation into human motor speech cortex. J Neurosci Meth. 2008;174:168-76.

5. Birbaumer N, Ghanayim N, Hinterberger T, et al. A spelling device for the paralysed. Nature. 1999;398:297-8.

6. Donoghue JP. Bridging the brain to the world: a perspective on neural interface systems. Neuron. 2008;60:511-21.

7. Kubler A, Nijboer F, Mellinger J, et al. Patients with ALS can use sensorimotor rhythms to operate a brain-computer interface. Neurology. 2005;64:1775-7.

8. Nijboer F, Sellers EW, Mellinger J, et al. A P300-based braincomputer interface for people with amyotrophic lateral sclerosis. Clin Neurophysiol. 2008;119:1909-16.

9. Sellers EW, Donchin E. AP300-based brain-computer interface: Initial tests by ALS patients. Clin Neurophysiol. 2006;117: 538-48.

10. Birbaumer N. Brain-computer-interface research: coming of age. Clin Neurophysiol. 2006;117:479-83.

11. Claussen J. Man, machine and in between. Nature. 2009;457(26): 1080-1.

12. Daly JJ, Wolpaw, JR. Brain-computer interfaces in neurological rehabilitation. Lancet Neurol. 2008;7:1032-43.

13. Del R Milan J, Carmena J. Invasive or noninvasive: understanding brain-machine interface technology. IEEE Eng Med Bio Mag. 2010;29(1):16-22.

14. Hatsopoulos NG, Donoghue JP. The science of neural interface systems. Annu Rev Neurosci. 2009;32:249-66.

15. Hochberg LR. Turning thought into action. N Engl J Med. 2008;359 (11):1175-7.

16. Lebedev MA, Nicolelis MAL. Brain-machine interfaces: past, present, and future. Trends Neurosci. 2006;29(9):536-46.

17. Leuthardt EC, Schalk G, Moran DW, Ojemann JG. The emerging world of motor neuroprosthetics: a neurosurgical perspective. Neurosurgery. 2006;59:1-14.

18. Leuthardt EC, Schalk G, Roland J, Rouse A, Moran DW. Evolution of brain-computer interfaces: going beyond classic motor physiology. Neurosurg Focus. 2009;27(1):E4.

19. Mussa-Ivaldi FA, Miller LE. Brain-machine interfaces: computational demands and clinical needs meet basic neuroscience. Trends Neurosci. 2003;26:329-34.

20. Nicolelis MAL. Actions from thoughts. Nature. 2001;409:403-7.

21. Nicolelis MAL. Brain-machine interfaces to restore motor function and probe neural circuits. Nature Rev Neurosci. 2003;4:417-22. 
22. Nicolelis MAL, Lebedev MA. Principles of neuronal ensemble physiology underlying the operation of brain-machine interfaces. Nat Rev Neurosci. 2009;10:530-40.

23. Normann RA. Technology insight: future neuroprosthetic therapies for disorders of the nervous system. Nature Clin Prac Neurol. 2007;3(8):444-52

24. Patil PG, Turner DA. The development of brain-machine interface neuroprosthetic devices. Neurotherapeutics. 2008;5:137-46.

25. Patil PG. Introduction: advances in brain-machine interfaces. Neurosurg Focus. 2009;27(1):E1

26. Ryu SI, Shenoy KV. Human cortical prostheses: lost in translation? Neurosurg Focus. 2009;27(1):E5:1-11.

27. Schwartz AB, Cui XT, Weber DJ, Moran DW. Brain-controlled interfaces: movement restoration with neural prosthetics. Neuron. 2006;52:205-20.

28. Scott SH. Cortical-based neuroprosthetics: when less may be more. Nature Neurosci. 2008;11(11):1245-6.

29. Wickelgren I. Tapping the mind. Science. 2003;299(5606):496-9.

30. Wolpaw JR, Birbaumer N, McFarland DJ, Pfurtscheller G, Vaughan TM. Brain-computer interfaces for communication and control. Clin Neurophys. 2002;113:767-91

31. Anderson NR, Wisneski K, Eisenman L, Moran DW, Leuthardt EC, Krusienski DJ. An offline evaluation of the autoregressive spectrum for electrocorticography. IEEE Trans Biomed Eng. 2009;56(3):913-16.

32. Georgopoulos AP, Schwartz AB, Kettner RE. Neuronal population coding of movement direction. Science. 1986;233:1416-19.

33. Pfurtscheller G, Neuper C, Guger C, et al. Current trends in Graz brain-computer interface (BCI) research. IEEE Trans Rehab Eng. 2000;8(2):216-19.

34. Ferris DP. The exoskeletons are here. J Neuroeng Rehab. 2009; 6:17.

35. Lynch CL, Popovic MR. Functional electrical stimulation. IEEE Contr Syst Mag. 2008;40-50.

36. Moritz, CT, Lucas TH, Perlmutter SI, Fetz EE. Forelimb movements and muscle responses evoked by microstimulation of cervical spinal cord in sedated monkeys. J Neurophysiol. 2007;97:110-20

37. Moritz CT, Perlmutter SI, Fetz EE. Direct control of paralysed muscles by cortical neurons. Nature. 2008;456:639-42.

38. Peckham PH, Knutson JS. Functional electrical stimulation for neuromuscular applications. Annu Rev Biomed Eng. 2005;7: $327-60$.

39. Pfurtscheller G, Muller GR, Pfurtscheller J, Gerner HJ, Rupp R. 'Thought' - control of functional electrical stimulation to restore hand grasp in a patient with tetraplegia. Neurosci Letters. 2003; 351:33-6.

40. Fetz EE, Finocchio DV. Operant conditioning of specific patterns of neural and muscular activity. Science. 1971;174:431-5.

41. Jarosiewicz B, Chase SM, Fraser GW, Velliste M, Kass RE, Schwartz AB. Functional network reorganization during learning in a brain-computer interface paradigm. Proc Nat Acad Sci. 2008;105(49): 19486-91.

42. Kennedy PR, Bakay RA. Restoration of neural output from a paralyzed patient by a direct brain connection. Neuroreport. 1998:9:1707-11.

43. Schmidt EM, McIntosh JS, Durelli L, Bak MJ. Fine control of operantly conditioned firing patterns of cortical neurons. Exp Neurol. 1978;61:349-69.

44. Buttfield A, Ferrez PW, Millan JR. Towards a robust BCI: error potentials and online learning. IEEE Trans Neural Sys Rehab Eng. 2006;14(2):164-8.

45. Nicolelis MAL, Ribiero S. Seeking the neural code. Sci Am. 295 2006;6:70-7.

46. Weber DJ, Stein RB, Everaert DG, Prochazka A. Decoding sensory feedback from firing rates of afferent ensembles recorded in cat dorsal root ganglia in normal locomotion. IEEE Trans Neur Sys Rehab Eng. 2006;14(2):240-3.

47. Vidal JJ. Real-time detection of brain events in EEG. Proc of the IEEE. 1977;65(5):633-41.

48. Pfurtscheller G, Cooper R. Frequency dependence of the transmission of the EEG from cortex to scalp. Electroencephalogr Clin Neurophysiol. 1975;38:93-6.
49. Buzsaki G. Rhythms of the brain. Oxford University Press; 2006.

50. Niedermeyer E, Lopes da Silva FH. The normal EEG of the waking adult. Baltimore: Williams and Wilkins; 1999.

51. Nunez PL, Srinivasan R. Electric fields of the brain: the neurophysics of EEG. Oxford University Press; 1981.

52. Guger C, Edlinger G, Harkam W, Niedermeyer I, Pfurtscheller G. How many people are able to operate an EEG-based braincomputer interface (BCI)? IEEE Trans Neural Sys Rehab Eng. 2003;11(2):145-7.

53. McFarland DJ, Krusienski DJ, Sarnacki WA, Wolpaw JR. Emulation of computer mouse control with a noninvasive braincomputer interface. J Neural Eng. 2008;5:101-10.

54. Min BK, Marzelli MJ, Yoo SS. Neuroimaging-based approaches in the brain-computer interface. Trends Biotech. 2010;28(11): 552-60.

55. Coyle S, Ward T, Markham C, McDarby G. On the suitability of near-infrared (NIR) systems for next-generation brain-computer interfaces. Physiological Measurement. 2004;25:815-22.

56. Sitaram R, Zhang H, Guan C, et al. Temporal classification of multichannel nearinfrared spectroscopy signals of motor imagery for developing a brain computer interface. Neuroimage. 2007:34:1416-27.

57. Gratton G, Fabiani M. Shedding light on brain function: the eventrelated optical signal. Trends Cog Sci. 2001;5(8):357-63.

58. Kennedy P, Andreasen D, Ehirim P, et al. Using human extracortical local field potentials to control a switch. J Neural Eng. 2004; $1: 72-7$

59. Crone NE, Miglioretti DL, Gordon B, et al. Functional mapping of human sensorimotor cortex with electrocorticographic spectral analysis: I Alpha and beta event-related desynchronization. Brain. 1998;121:2271-99.

60. Crone NE, Miglioretti DL, Gordon B, Lesser RP. Functional mapping of human sensorimotor cortex with electrocorticographic spectral analysis. II. Event-related synchronization in the gamma band. Brain. 121:2301-15.

61. Leuthardt EC, Schalk G, Wolpaw JR, Ojemann JG, Moran DW. A brain-computer interface using electrocorticographic signals in humans. J Neural Eng. 2004;1:63-71.

62. Leuthardt EC, Freudenberg Z, Bundy D, Roland J. Microscale recordings from human motor cortex: implications for minimally invasive electrocortigraphic brain-computer interfaces. Neurosurg Focus. 2009;27(1):E10,1-8.

63. Yuen TG, Agnew WF, Bullara LA. Tissue response to potential neuroprosthetic materials implanted subdurally. Biomaterials. 1987;8:138-41

64. Slutzky MW, Jordan LR, Krieg T, Chen M, Mogul DJ, Miller LE. Optimal spacing of surface electrode arrays for brain-machine interface applications. J Neural Eng. 2010;7(2):26004-12.

65. Kozelka JW, Pedley TA. Beta and mu rhythms. J Clin Neurophysiol. 1990;7:191-207.

66. Pfurtscheller G, Graimann B, Huggins JE, Levine SP, Schuh LA. Spatiotemporal patterns of beta desynchronization and gamma synchronization in corticographic data during self-paced movement. Clin Neurophysiol. 2003;114:1226-36.

67. Singer W. Synchronization of cortical activity and its putative role in information processing and learning. Annu Rev Physiol. 1993;55:349-74.

68. Womelsdorf T, Schoffelen JM, Oostenveld R, et al. Modulation of neuronal interactions through synchronization. Science. 2007; 316:1609-12.

69. Leuthardt EC, Kim W, Anderson N, Wisneski K, Barbour D, Schalk G. Decoding speech phonemes from Broca's area for neuroprosthetic application. Neurosurgery. 2008;62(6):1418.

70. Schalk G, Miller KJ, Anderson NR, et al. Two-dimensional movement control using electrocorticographic signals in humans. J Neural Eng. 2008;5:75-84.

71. Kennedy PR, Bakay RAE, Moore MM, Adams K, Goldwaithe J. Direct control of a computer from the human central nervous system. IEEE Trans Rehab Eng. 2000;8(2):198-202.

72. Mulliken GH, Musallam S, Andersen RA. Decoding trajectories from posterior parietal cortex ensembles. J Neurosci. 2008;28 (48): 12913-26. 
73. Schwartz AB, Kettner RE, Georgopoulos AP. Primate motor cortex and free arm movements to visual targets in three-dimensional space. I. Relations between single cell discharge and direction of movement. J Neurosci. 1988;8(8):2913-27.

74. Rodriguez-Oroz MC, Rodriguez M, Guridi J, et al. The subthalamic nucleus in Parkinson's disease: Somatotopic organization and physiological characteristics. Brain. 2001;124:1777-90.

75. Donoghue JP. Connecting cortex to machines: recent advances in brain interfaces. Nature Neurosci. 2002;5:1085-8.

76. Laubach M, Wessberg J, Nicolelis MAL. Cortical ensemble activity increasingly predicts behaviour outcomes during learning of a motor task. Nature. 2000;405:567-71

77. Taylor DM, Tillery SIH, Schwartz AB. Direct cortical control of 3D neuroprosthetic devices. Science. 2002;296:1829-32.

78. Wessberg J, Stambaugh CR, Kralik JD, et al. Real-time prediction of hand trajectory by ensembles of cortical neurons in primates. Nature. 2000;408:361-5.

79. Veliste M, Perel S, Spalding MC, Whitford AS, Schwartz AB. Cortical control of a prosthetic arm for self-feeding. Nature. 2008:453:1098-101.

80. Hochberg LR, Serruya MD, Friehs GM, et al. Neuronal ensemble control of prosthetic devices by a human with tetraplegia. Nature. 2006;442:164-71

81. Polikov VS, Tresco PA, Reichert WM. Response of brain tissue to chronically implanted neural electrodes. J Neurosci Meth. 2005; $148: 1-18$

82. Shain W, Spataro L, Dilgen J, et al. Controlling cellular reactive responses around neural prosthetic devices using peripheral and local intervention strategies. IEEE Trans Neural Syst Rehab Eng. 2003;11:186-8

83. Szarowski DH, Andersen MD, Retterer S, et al. Brain responses to micro-machined silicon devices. Brain Res. 2003;983:23-35.

84. Williams JC, Rennaker RL, Kipke DR. Long-term neural recording characteristics of wire microelectrode arrays implanted in cerebral cortex. Brain Res Protocols. 1999;4:303-13.

85. Pang C, Tai YC, Burdick JW, Andersen RA. Electrolysis-based parylene balloon actuators for movable neural probes. Proc of 2nd IEEE International Conference on Nano/Micro Engineered and Molecular Systems, Jan 16-19, 2007;913-16.

86. Riegar R, Taylor JT. Design strategies for multi-channel low-noise recording systems. Analog Integr Circ Sig Process. 2009;58: 123-33.

87. Rizk M, Bossetti CA, Jochum TA, et al. A fully implantable 96channel neural data acquisition system. J Neural Eng. 2009;6: $1-14$.

88. Sarpeshkar R, Wattanapanitch W, Arfin SK, et al. Low-power circuits for brain-machine interfaces. IEEE Trans Biomed Circ Sys. 2008;2(3): 173-82.

89. Pesaran B, Musallam S, Andersen RA. Cognitive neural prosthetics. Current Biol. 2006;16(3):R77-80.

90. Rickert J, Oliveira SC, Vaadia E, Aertsen A, Rotter S, Mehring C. Encoding of movement direction in different frequency ranges of motor cortical local field potentials. J Neurosci. 2005;25: 8815-24.

91. Pesaran B. Uncovering the mysterious origins of local field potentials. Neuron. 2008;61:1-2.

92. Andersen RA, Musallam S, Pesaran B. Selecting the signals for a brain-machine interface. Curr Opin Neurobiol. 2004;14:1-7.

93. Mehring C, Rickert J, Vaadia E, Cardoso de Oliviera S, Aertsen A, Rotter S. Inference of hand movements from local field potentials in monkey motor cortex. Nature Neurosci. 2003;6 (12): $1253-4$

94. Murthy VN, Fetz EE. Synchronization of neurons during local field potential oscillations in sensorimotor cortex of awake monkeys. J Neurophysiol. 1996;76:3968-82.

95. Pesaran B, Pezaris J, Sahani M, Mitra PM, Andersen RA. Temporal structure in neuronal activity during working memory in macaque parietal cortex. Nat Neurosci. 2002;5:805-11.

96. Zhuang J, Truccolo W, Vargas-Irwin C, Donoghue JP. Decoding 3$\mathrm{D}$ reach and grasp kinematics from high-frequency local field potentials in primate motor cortex. IEEE Trans Biomed Engi. 2010;57(7):1774-84
97. Katzner S, Nauhaus I, Benucci A, Bonin V, Ringach DL, Carandini M. Local origin of field potentials in visual cortex. Neuron. 2009;61:35-41.

98. Scherberger H, Jarvis MR, Andersen RA. Cortical local field potential encodes movement intentions in the posterior parietal cortex. Neuron. 2005;46:347-54.

99. Kellis SS, House PA, Thomson KE, Brown R, Greger B. Human neocortical electrical activity recorded on nonpenetrating microwire arrays: applicability for neuroprostheses. Neurosurg Focus. 2009;27(1):E9

100.Kakei S, Hoffman DS, Strick PL. Muscle and movement representations in the primary motor cortex. Science. 1999;285: 2136-9.

101. Stark E, Drori R, Abeles M. Motor cortical activity related to movement kinematics exhibits local spatial organization. Cortex. 2009;45:418-31.

102. Scott SH. Optimal feedback control and the neural basis of volitional motor control. Nat Rev Neurosci. 2004;5:532-46.

103. Rathelot JA, Strick PL. Muscle representation in the macaque motor cortex: an anatomical perspective. Proc Nat Acad Sci. 2006;103(21):8257-62.

104. Georgopoulos AP. Higher order motor control. Ann Rev Neurosci. 1991;14:361-77.

05. Moran DW, Schwartz AB. Motor cortical representation of speed and direction during reaching. J Neurophysiol. 1999;82: 2676-92.

106. Yanagisawa T, Hirata M, Saitoh Y, et al. Neural decoding using gyral and intrasulcal electrocorticograms. Neuroimage. 2009;45: 1099-106.

107. Andersen RA, Hwang EJ, Mulliken GH. Cognitive neural prosthetics. Annu Rev Psychol. 2010;61:169-90.

108. Cohen YE, Andersen RA. A common reference frame for movement plans in the posterior parietal cortex. Nat Rev Neurosci. 2002;3:553-62.

109. Musallam S, Corneil BD, Greger B, Scherberger H, Andersen RA. Cognitive control signals for neural prosthetics. Science. 2004; 305:258-62.

110. Rizzuto DS, Mamelak AN, Sutherling WW, Fineman I, Andersen RA. Spatial selectivity in human ventrolateral prefrontal cortex. Nat Neurosci. 2005;8(4):415-17.

111. Snyder LH, Batista AP, Andersen RA. Coding of intention in the posterior parietal cortex. Nature. 1997;386:167-70.

112. Ramsey NF, van de Heuvel MP, Kho KH, Leijten FSS. Towards human BCI applications based on cognitive brain systems: an investigation of neural signals recorded from the dorsolateral prefrontal cortex. IEEE Trans Neur Sys Rehab Eng. 2006; 214-17.

113. Campos M, Breznen B, Bernheim K, Andersen RA. Supplementary motor area encodes reward expectancy in eye-movement tasks. J Neurophysiol. 2005;94:1325-35.

114. Cisek P, Kalaska JF. Neural correlates of mental rehearsal in dorsal premotor cortex. Nature. 2004;431:993-6.

115. Kalaska JF, Crammond DJ. Cerebral cortical mechanisms of reaching movements. Science. 1992;255(5051):1517-23.

116. Rizzolatti G, Fogassi L, Gallese V. Motor and cognitive functions of the ventral premotor cortex. Curr Opin Neurobiol. 2002;12:149-54

117. Santhanam G, Ryu SI, Yu BM, Afshar A, Shenoy KV. A high performance brain-computer interface. Nature. 2006;442:195-8.

118. Tankus A, Yeshurun Y, Flash T, Fried I. Encoding of speed and direction of movement in the human supplementary motor area. J Neurosurg. 2009;110:1304-16.

119. Kandel ER, Schwartz JH, Jessell TM. Principles of Neural Science. 3rd Ed. E Norwalk, Conneticut: Appleton \& Lange; 1991.

120. Amador N, Fried I. Single-neuron activity in the human supplementary motor area underlying preparation for action. $\mathrm{J}$ Neurosurg. 2004;100:250-9.

121. Paradiso G, Saint-Cyr JA, Lozano AM, Lang AE, Chen R. Involvement of the human subthalamic nucleus in movement preparation. Neurol. 2003;61:1538-45.

122. Paradiso G, Cunic D, Saint-Cyr JA, et al. Involvement of human thalamus in the preparation of self-paced movement. Brain. 2004:127:2717-31. 
123.Purzner J, Paradiso G, Cunic D. Involvement of the basal ganglia and cerebellar motor pathways in the preparation of self-initiated and externally triggered movements in humans. J Neurosci. 2007;27(22):6029-36.

124. Patil PG, Carmena JM, Nicolelis MAL, Turner DA. Ensemble recordings of human subcortical neurons as a source of motor control signals for a brain-machine interface. Neurosurgery. 2004;55(1):27-38

125. Adachi Y, Kawai J, Miyamoto M, et al. IEEE Trans App Supercond. 2009;19(3):861-6.

126. Kawabata S, Komori H, Mochida K, Ohkubo H, Shinomiya H. Visualization of conductive spinal cord activity using a biomagnetometer. Spine. 2003;27:475-9.

127. Stein RB, Mushahwar V. Reanimating limbs after injury or disease. Trends Neurosci. 2005;28(10):518-24.

128. Vogelstein RJ, Tenore FVG, Guevremont L, Etienne-Cummings R, Mushahwar VK. A silicon central pattern generator controls locomotion in vivo. IEEE Trans Biomed Circ Sys. 2008;2(3): 212-22.

129. Peckham PH, Kilgore KL, Keith MW, Bryden AM, Bhadra N, Montague FW. An advanced neuroprosthesis for restoration of hand and upper arm control using an implantable controller. J. Hand Surg. 2002;27A:265-76.

130. Wilkinson HA. Hope, false hope, and self-fulfilling prophecy. Surg Neurol. 2005;63(1):84-6.

131. Birbaumer N. Breaking the silence: brain-computer interfaces (BCI) for communication and motor control. Psychophysiology. 2006;43:517-32.

132. Neumann N, Kubler A. Training locked-in patients: a challenge for the use of brain-computer interfaces. IEEE Trans Neur Sys Rehab Engin. 2003;11(2):169-72.

133. Donoghue JP, Nurmikko A, Black M, Hochberg LR. Assistive technology and robotic control using motor cortex ensemblebased neural interface systems in humans with tetraplegia. J Physiol. 2007;579(3):603-11.

134. ClinicalTrials.gov [Internet]. Braingate2: feasibility study of an intracortical neural interface system for persons with tetraplegia. [cited 2010 Aug 7]. Available from: http://www.clinical trials.gov/ct2/show/NCT00912041
135.Kempf Katalavox [Internet]. Voice activated power wheelchair and other devices for quadriplegics. [cited 2010 Aug 7]. Available from: http://www.katalavox.com/wheelch1.htm

136. Taylor TN, Davis PH, Torner JC, Holmes J, Meyer JW, Jacobson MF. Lifetime cost of stroke in the United States. Stroke. 1996; 27:1459-66.

137. Duncan PW, Goldstein LB, Horner RD, Landsman PB, Samsa GP, Matchar DB. Similar motor recovery of upper and lower extremities after stroke. Stroke. 1994;25:1181-8.

138. Buch E, Weber C, Cohen LG, et al. Think to move: a neuromagnetic brain-computer interface (BCI) system for chronic stroke. Stroke. 2008;39:910-17.

139. Wisneski KJ, Anderson N, Schalk G, Smyth M, Moran D, Leuthardt EC. Unique cortical physiology associated with ipsilateral hand movements and neuroprosthetic implications. Stroke. 2008;39: 3351-9.

140. Al-Otaibi FAJ, Alabousi A, Burneo JG, Lee DH, Parrent AG, Steven DA. Clinically silent magnetic resonance imaging findings after subdural strip electrode implantation. J Neurosurg. 2010;112:461-6.

141. Placantonakis DG, Shariff S, Lafaille F, et al. Bilateral intracranial electrodes for lateralizing intractable epilepsy: efficacy, risk, and outcome. Neurosurgery. 2010;66(2):274-83.

142. Voges J, Waerzeggers Y, Maarour M, et al. Deep-brain stimulation: long-term analysis of complications caused by hardware and surgery - experiences from a single center. J Neurol Neurosurg Psychiatry. 2006;77:868-72.

143. Farwell LA, Donchin E. Talking off the top of your head: toward a mental prosthesis utilizing event-related brain potentials. Electroencephalogr Clin Neurophysiol. 1988;70:510-23.

144. Wolpaw JR, McFarland DJ. Control of a two-dimensional movement signal by a noninvasive brain-computer interface in humans. PNAS. 2004;101(51): 17849-54.

145. Anderson K. Targeting recovery: priorities of the spinal cordinjured population. J Neurotrauma. 2004;21(10):1371-83. 